
\title{
Research S Suare fere \\ Pain Diary in Bands Based on SBAR Communication Mode Application of Nursing Management in Patients with Postherpetic Neuralgia
}

Ping Lin

Fushun people's Hospital

Guo Mu

Affiliated Hospital of Southwest Medical University

Cehua Ou

Affiliated Hospital of Southwest Medical University

liang yu ( $\triangle$ painyuliang@126.com)

Affiliated Hospital of Southwest Medical University

\section{Research Article}

Keywords: Pain Diary, SBAR, Postherpetic neuralgia, Self-assessment

Posted Date: December 2nd, 2020

DOl: https://doi.org/10.21203/rs.3.rs-109920/v1

License: (c) (i) This work is licensed under a Creative Commons Attribution 4.0 International License.

Read Full License 


\section{Abstract}

Pain diary shows an important role in the pain management of patients with chronic cancer pain, but it has not been mentioned in the nursing management of Postherpetic neuralgia (PHN). This study explored the impact of pain diary on patients with postherpetic neuralgia during hospitalization. Patients with postherpetic neuralgia were randomly divided into a pain diary group and a control group. During the hospitalization, the diary group was instructed to record the pain diary, and the control group was given routine care. Evaluating VAS score of the patients for 10 days on admission, Thai Brief Pain questionnaire score during mid-hospitalization, and the amount and satisfaction of the patient's condition information collected upon discharge. On the third day after admission, the VAS score of the diary group was significantly lower than that of the control group. The Thai Brief Pain results showed that the pain diary group had better mood, sleep and enjoyment of life than the control group. The number of patient's condition information from the diary group was significantly more than the control group, the diary group had better satisfaction with nurses and doctors than the control group. The short-term pain diary should have a better role in PHN hospitalization.

\section{Introduction}

Timely assessment of patient pain is very important for effective pain control, especially for patients with $\mathrm{PHN}$, which is often combined with hyperalgesia and explosive pain ${ }^{1,2}$. Lack of pain assessment will lead to insufficient pain control, affecting treatment results and subjective feelings of patients ${ }^{3}$. Although we know the importance of pain assessment, it is difficult for clinical nurses and doctors to dynamically assess the pain of patients with limited medical human resources. Pain self-assessment tools, such as pain diaries, have shown good effects in the management of cancer-related pain ${ }^{4-7}$ allowing patients to timely reflect the degree, nature and relief of pain when it occurs. This method proved to be highly reliable ${ }^{8}$. But carefully assessing patient pain diaries and extracting key information often increases the workload of busy clinicians, and nurses using the SBAR model can just make up for this deficiency. The SBAR model is a standardized and structured communication model proposed by the World Health Organization (WHO $)^{9}$, which consists of four parts: Situation, Background, Assessment, and Recommendation. Theoretically, the use of pain diary by nurses and doctors in SBAR mode can better grasp the patient's pain situation in a timely and accurate manner and handle it appropriately ${ }^{10}$. Studies of pain diary for pain management in patients with $\mathrm{PHN}$ have not yet been seen囚Therefore, our research explored the use of pain diary in SBAR mode for pain management during hospitalization of PHN patients and theorized that the use of pain diary can improve the pain situation of this population.

\section{Methods}

\section{Study design and participants}

The current study is a single-center randomized controlled trial following CONSORT 2010 clinical trial guidelines ${ }^{11}$ to explore the role of pain diary in pain management during hospitalization for PHN patients. 
This study was managed and supervised by the Ethics Committee of the Affiliated Hospital of Southwest Medical University (KY2019073) and the protocol of this study was registered at the Chinese Clinical Trial Registry (www.chictr.org.cn) on 07/03/2020 (Registration number: ChiCTR2000030565). The protocol of current study can be seen at supplementary material (Supplementary.1). All participants signed informed consent, and was diagnosed with PHN, aged 18-70 years, patients with clear intelligence and correct writing were included in this study. Patients with PHN less than 10 days in hospital and baseline VAS pain scores less than 3 were excluded.

\section{Sample size}

Since there is no data on pain diary in the pain management of PHN patients, the sample size calculation is based on a previous cancer-related pain study. The pain score in the pain diary and the control group was $2.76(S D=1.64)$ and $2.15(S D=1.67)$. The sample size ratio was $1: 1$, $a$ error 0.05 and $\beta$ error 0.2 , and effect size $36 \%$. Considering that the subjects were hospitalized patients, we set the lost follow-up rate to $5 \%$. The final calculation required 59 people per group.

\section{Procedure}

Prior to randomization, all patients were interviewed to record VAS scores to determine baseline pain levels, assess the location and nature of pain, and record demographic characteristics. For patients who met the inclusion criteria, after signing the informed consent, they were randomly divided into the diary group and the control group using computer-generated random numbers. Diary group and control group patients are allocated in different wards to avoid interference. As it involves the guidance of patients in the diary group, the blind method cannot be adopted in this study. However, the final data collection and statistical analysis are performed by people who do not know the grouping situation.

All patients were instructed on admission how to describe their pain on a scale of 0 to 10 , with 0 being painless and 10 being excruciatingly painful. Trained pain nurses conducted daily dynamic selfassessment of pain in the diary group and kept a pain diary, mainly recording the cause, degree and relief of pain, whether to seek help and some questions about pain treatment (Table.1). Patients in the diary group were interviewed the first two days to ensure that they understood the correct self-assessment of pain and kept a pain diary. Patients were told not to keep a diary when they need urgent help and to call a nurse or doctor immediately. Patients in the control group received routine pain care and were asked about their disease information during the daily ward rounds.

After receiving the pain diary, the nurses proceeded according to the SBAR model. Establish a uniform transfer system between doctors and nurses, and describe patient conditions and problems in a unified and standardized manner. The nurse extracts pain diary information, conducts a preliminary assessment of current problems, and distinguishes between problems that nursing can solve and problems that need to be addressed by a doctor. After processing the patient's needs in a timely manner, the information is fed back to the doctor in accordance with the SBAR communication method, and the doctor is adjusted to the treatment. 


\section{Data collection}

The basic information of the enrollees of this trial was collected by the nurses in the inpatient department before randomization, including the patient's gender, age, baseline pain level, pain site, education level. The average daily pain level of the patients, the completion of the Thai-BPI questionnaire and the patient satisfaction survey were completed by a third-party hospital researcher. The trial group assigned them to hide. When assessing pain levels daily, patients were invited to interview rooms individually. The evaluators explained to them the VAS standard and how to describe the degree of pain, and then the patients reported their pain levels for the past day on their own. This assessment was performed only once a day. The third-party evaluator invited the patient to fill in the Thai-BPI questionnaire on the fifth day after the patient was admitted to the hospital. The information extraction of pain diary is performed by trained nurses. They collect the patient's pain diary every day and carefully read the extracted diary information to record the disease information and pain information fed back by the patient. And all the data was filled in using an electronic form. The data was stored in the clinic of the hospital. Research database.

\section{Measures}

Daily pain level. VAS score was used to assess the pain level of patients in both groups during daily ward rounds (as described above) for 10 consecutive days. Determine the patient's overall pain on a daily basis.

Thai-BPI. The Thai-BP questionnaire is an assessment tool for assessing the pain and physical condition of patients ${ }^{12}$, which can reflect the pain of patients for a period of time. It can assess the intensity of pain in patients with the most severe pain, the slightest pain, and general pain. In addition, it also includes a 24-hour average pain intensity, with pain levels ranging from 0 to 10; 0 means no pain and 10 means unbearable pain. There are 13 entries, each with a score of 10 points. The higher the score, the worse the situation. We only selected 10 entries that are closely related to our research. Patients were invited to a Thai-BP questionnaire interview at mid-term.

Number of patient's condition information.Pain diaries for patients in the diary group were organized by the nursing staff each day, and the patient's feedback information includes the cause, extent, remission, and treatment effect of pain were recorded. In addition, the condition information reported by the two groups during the morning round were recorded. If the questions obtained during the rounds are similar to those in the diary, they are removed and recorded as only one information. At any time, the condition information that the patient reports directly to the attending doctor or nurse will also be recorded, and the recording principle is the same as before. Finally calculate the total number of information for each patient within 10 days.

Patient satisfaction. On the 10th day, the patient's satisfaction with the nurses and doctors was evaluated. On a scale of 0 to 10, 0 was bad and dissatisfied, and 10 was most satisfied. Patients included need to be evaluated separately for nurses and attending physicians. In addition, in the interview on the 
last day, the patients in the diary group were asked if the pain diary was good for their pain, and the patients could answer that it was very beneficial, with some benefits and no benefits.

\section{Statistical analysis}

Data entry and sorting are done in Microsoft Excel-2016 software; Data analysis was performed using IBM SPSS version 24.0. Quantitative data conforming to the normal distribution are expressed by mean and standard deviation, and non-normal distribution data are expressed by interquartile range. For the comparison of qualitative data such as demographic characteristics, the chi-square test and Fisher exact probability method were used for statistical description; Comparison of pain scores within the group was performed using one-way analysis of variance (ANOVA); Comparison of pain scores between groups, Thai-BPI scores of the two groups of patients, number of patient's condition information, and satisfaction comparison were performed using Student's $t$ test. Statistical result $P<0.05$ indicates that the difference is statistically significant.

\section{Results}

\section{Demographic information}

A total of $135 \mathrm{PHN}$ patients were recruited in this study from July 2019 to March 2020, but 16 patients were not included in the final statistical analysis. Of these 16 patients, 2 were hospitalized for less than 10 days, 1 patient had to be transferred to another department for treatment due to heart disease, and 8 patients withdrew during the trial, 5 others do not meet the inclusion criteria. The remaining 119 patients were randomly assigned to the diary group and the control group, 60 and 59 respectively (Fig.1). Most of the patients in the two groups were females. Most of the previous sites of herpes zoster were located in the thoracic ribs. There was no significant difference in the average age, initial pain score and education level of the patients (Table.2).

\section{Pain diary can relieve patients' pain to some extent}

Patients in both groups were interviewed for 10 consecutive days after admission, and their average daily pain intensity was assessed using the VAS method. All patients completed 10 consecutive days of interviews and pain assessments (Completion rate 100\%, completion rate is defined as the number of patients who get accurate and true divided by the number of patients who should theoretically be evaluated in each group). On the 4th day after admission, the VAS scores of both groups were significantly lower than when they were admitted, which may be related to patients receiving systemic treatment ( $P \otimes 0.05)$; while from the 3rd day after admission, the pain score in the diary group was significantly lower than that in the control group (Fig.2).

\section{Results of Thai-BPI questionnaire during mid-hospitalization}

Two groups of patients accepted the Thai-BPI questionnaire on the fifth day after admission. Among them, 55 patients in the diary group completed the questionnaire (91.6\%). The remaining 5 were not 
standardized and refused to complete again. 57 people in the control group completed filling in (96.6\%), 2 people refused to fill in. The results showed that there was no significant difference in the maximum pain, the minimum pain, and the therapeutic relief pain between the two groups of patients. The diary group had better average pain scores, mood, sleep status, and enjoyment of life than the control group (P凶0.05). More details are shown in (Table. 3 ).

\section{Pain diary helps to get more patient information and improve patient satisfaction}

After comparing the number of patients' information collected during the 10 days after admission to the two groups, we found that the diary group was significantly more than the control group. The average number of disease information collected in the diary group was $55.2(S D=2.3)$ and the control group was $37(S D=3.5)$. In the diary group, $56.3 \%$ of the condition information was timely feedback and processed by the doctor, $40.0 \%$ was performed by the nurse, and the remaining $3.7 \%$ could not be processed; in the control group, $54.0 \%$ was processed by the doctor, $32.4 \%$ was processed by the nurse, and the remaining $13.6 \%$ were not processed (Fig.3A). Interestingly, diary patients were significantly more satisfied with caregivers and doctors than the control group (Fig.3B). Interestingly, 42(70.0\%) of the patients in the diary group felt that the pain diary was very helpful for their pain management, $14(23.3 \%)$ felt that there were some benefits, and the remaining 4 (6.70\%) considered no benefit.

\section{Discussion}

Patients 'timely feedback on pain intensity plays an important role in pain treatment ${ }^{13,14}$. In the lagging pain assessment, patients' memories of pain often have deviations. In addition, during the short daily communication, doctors and nurses have limited patient information. Patients should play a more active self-assessment of pain during hospitalization ${ }^{15}$. Among the various pain assessment methods, pain self-reporting is emphasized as the most reliable method ${ }^{16}$. Pain diary is a dynamic and continuous method of patient self-reporting ${ }^{17}$. Many studies have shown good results in the management of cancer pain. In addition, it also has a positive effect in the treatment of some non-cancerous pains, such as lower back pain ${ }^{18,19}$. However, the current application of pain diary is to explore the role of chronic pain management. The effect of diary of short time use on recovery from PHN is unknown. Can't help asking whether the pain diary can also have a positive effect on pain control during short-term hospitalizations for patients with neuropathic pain.

In this study, we explored the effect of pain diary on the intensity of pain in patients with PHN during hospitalization. The results show that the pain diary helps to reduce the pain level during the hospital stay and also helps to obtain more information about the patient's condition. These are positive for pain control. In addition, pain diary also played a positive role in improving the satisfaction of patients with PHN during hospitalization. The completion rate and recognition of the diaries in the diary group in this study were relatively high. These results validate our hypothesis that pain diary also have a positive role in pain management during short-term hospitalizations for patients with $\mathrm{PHN}$. 
PHN is a type of stubborn neuropathic pain, which is characterized by severe pain, often with hyperalgesia, and changes in pain intensity. Continuous pain monitoring difficult for PHN patients during hospitalization. Physicians are often managers of patients recalling pain when assessing their pain. deviations are unavoidable. In addition, it is challenging to obtain the patient's condition in a short-term communication. To avoid issues related to retrospective self-reporting, researchers and clinicians turn to diaries for data on patients who are closer to events or experiences of interest. The assessment of pain diaries serves to guide physicians in their choice of treatment strategy. In clinical trials, they are often used to determine whether therapeutic interventions are effective ${ }^{18,20}$. In order to allow the information from the pain diary to be properly processed, we introduced the SBAR mode, in which nurses extract the diary content in time, make preliminary assessments, sort out information, and assist doctors in processing.

We analyzed why pain journals can benefit short-term hospitalized PHN patients. First of all『pain diary is a symptom management method for patients, They can perform self-assessment in time when pain occurs, and accurate pain information is critical for treatment. Although there are some opinions that excessive attention to pain may cause patients' psychological burden and even aggravate pain ${ }^{21}, \mathrm{PHN}$ is not persistent pain. Most of them are induced pain. The seizure law is difficult to understand, so the patient's self-attention is more conducive to pain control. Second, the patient's pain diary adds information feedback, helping nurses and doctors to have a more comprehensive grasp of pain information, timely adjustment of treatment methods and understanding of the effect of treatment, and timely resolution of patient discomfort can greatly increase patient trust. These are all can bring benefits

to pain control. In the end, we believe that the introduction of SBAR mode has increased the accuracy and efficiency of treating patients' conditions. The application of SBAR mode can reduce the deviation of doctor and nurse handover, and the information transmission is more accurate.

Of course, this study still has some limitations. This study did not conduct secondary analysis for factors such as education level, comorbidities, etc. The sample size of this study is small and it is a single-center study. Large samples and multi-center clinical trials may still be needed.

\section{Conclusion}

Through this study, we found that pain diaries are beneficial for pain control during PHN patients' hospitalization and can increase patient satisfaction, which is helpful to improve the management comfort of PHN. This study shows that short-term use of pain diary has several episodes during the treatment of PHN, but whether it is equally effective for other pain-related diseases may require further research.

\section{Declarations}

\section{Compliance with Ethical Standards}




\section{Funding}

This study has not received any funding.

\section{Conflict of Interest}

The authors declare that they have no conflict of interest.

\section{Ethical Approval}

This study was proved by the Affiliated Hospital of Southwest Medical University Ethics Committee. This material is original and has not been published in whole or in part elsewhere.

\section{Informed Consent}

Informed written consent was obtained from the patients.

\section{Author Contributions}

P.L. designed the study, performed experiments, prepared figures, prepared the manuscript. G.M. designed the study, performed experiments, analyzed data and prepared figures. C.O. performed part of the experiments and analyzed data. L.Y. designed the study, accepts responsibility for conduct of research and final approval.

\section{References}

1. Johnson RW and Rice AS. Clinical practice: postherpetic neuralgia. N Engl J Med . 371,1526-1533 (2014).

2. Kato J, Matsui N, Kakehi Y, Murayama E, Ohwada S \& Sugihara M. Mirogabalin for the management of postherpetic neuralgia: a randomized, double-blind, placebo-controlled phase 3 study in Asian patients. Pain. 160, 1175-1185 (2019).

3. AlMazrou SH, Elliott RA, Knaggs RD \& AlAujan SS. Cost-effectiveness of pain management services for chronic low back pain: a systematic review of published studies. BMC health services research 2020; 20, 194 (2020).

4. Stinson JN, Stevens BJ, Feldman BM, Streiner D, McGrath PJ, Dupuis A, Gill N \& Petroz GC. Construct validity of a multidimensional electronic pain diary for adolescents with arthritis. Pain. 2008. 136 281-292 (2008).

5. Schumacher KL, Koresawa S, West C, Dodd M, Paul SM, Tripathy D, Koo P \& Miaskowski C. The usefulness of a daily pain management diary for outpatients with cancer-related pain. Oncology nursing forum. 29,1304-1313 (2002).

6. Charoenpol FN, Tontisirin N, Leerapan B, Seangrung R \& Finlayson RJ. Pain experiences and intrapersonal change among patients with chronic non-cancer pain after using a pain diary: a mixed- 
methods study. J Pain Res. 12, 477-487 (2019).

7. Gaertner J, Elsner F, Pollmann-Dahmen K, Radbruch L \& Sabatowski R. Electronic pain diary: a randomized crossover study. J Pain Symptom Manage. 28, 259-267 (2004).

8. Schumacher KL, Koresawa S, West C, Dodd M, Paul SM, Tripathy D, Koo P \& Miaskowski C. The usefulness of a daily pain management diary for outpatients with cancer-related pain. Oncol Nurs Forum. 29, 1304-1313 (2002).

9. Uhm JY, Ko Y and Kim S. Implementation of an SBAR communication program based on experiential learning theory in a pediatric nursing practicum: A quasi-experimental study. Nurse Educ Today.80, 78-84 (2019).

10. MacDougall-Davis SR, Kettley L and Cook TM. The 'go-between' study: a simulation study comparing the 'Traffic Lights' and 'SBAR' tools as a means of communication between anaesthetic staff. Anaesthesia. 71, 764-772 (2016).

11. Schulz KF, Altman DG, Moher D and CONSORT Group. CONSORT 2010 statement: updated guidelines for reporting parallel group randomised trials. PLoS Med. 7, e1000251 (2010).

12. Keawnantawat $P$, Thanasilp $S$ and Preechawong $S$. Translation and Validation of the Thai Version of a Modified Brief Pain Inventory: A Concise Instrument for Pain Assessment in Postoperative Cardiac Surgery. Pain Pract. 17, 763-773 (2017).

13. de Wit R, van Dam F, Hanneman M, Zandbelt L, van Buuren A, van der Heijden K, Leenhouts G, Loonstra $\mathrm{S}$ and Abu-Saad HH. Evaluation of the use of a pain diary in chronic cancer pain patients at home. Pain . 79, 89-99 (1999).

14. Dirk K, Rachor GS and Knopp-Sihota JA. Pain Assessment for Nursing Home Residents: A Systematic Review Protocol. Nurs Res. 68, 324-328 (2019).

15. Lucas R, Zhang Y, Walsh SJ, Evans H, Young E and Starkweather A. Efficacy of a Breastfeeding Pain Self-Management Intervention: A Pilot Randomized Controlled Trial. Nurs Res. 68, E1-E10 (2019).

16. Brunkert T, Simon M, Ruppen W and Zúñiga F. Pain Management in Nursing Home Residents: Findings from a Pilot Effectiveness-Implementation Study. J Am Geriatr Soc. 67, 2574-2580 (2019).

17. Dampier C, Ely B, Brodecki D and O'Neal P. Characteristics of pain managed at home in children and adolescents with sickle cell disease by using diary self-reports. Pain . 3, 461-470 (2002).

18. Ferrari R. Effect of a pain diary use on recovery from acute low back (lumbar) sprain. Rheumatol Int. 35, 55-59 (2015).

19. Charoenpol FN, Tontisirin N, Leerapan B, Seangrung R and Finlayson RJ. Pain experiences and intrapersonal change among patients with chronic non-cancer pain after using a pain diary: a mixedmethods study. J Pain Res. 12, 477-487 (2019).

20. Ferrari R and Louw D. Effect of a pain diary use on recovery from acute whiplash injury: a cohort study. J Zhejiang Univ Sci B. 14, 1049-1053 (2013).

21. Kasahara S, Niwa SI, Matsudaira K, Sato N, Oka H and Yamada Y. Attention deficit hyperactivity disorder and chronic pain. Psychosom Med 2020. DOI: 10.1097/psy.0000000000000789. 


\section{Tables}

Table.1 Schematic diagram of pain diary (English language version).

Date:

\section{PAIN DǍIRY}

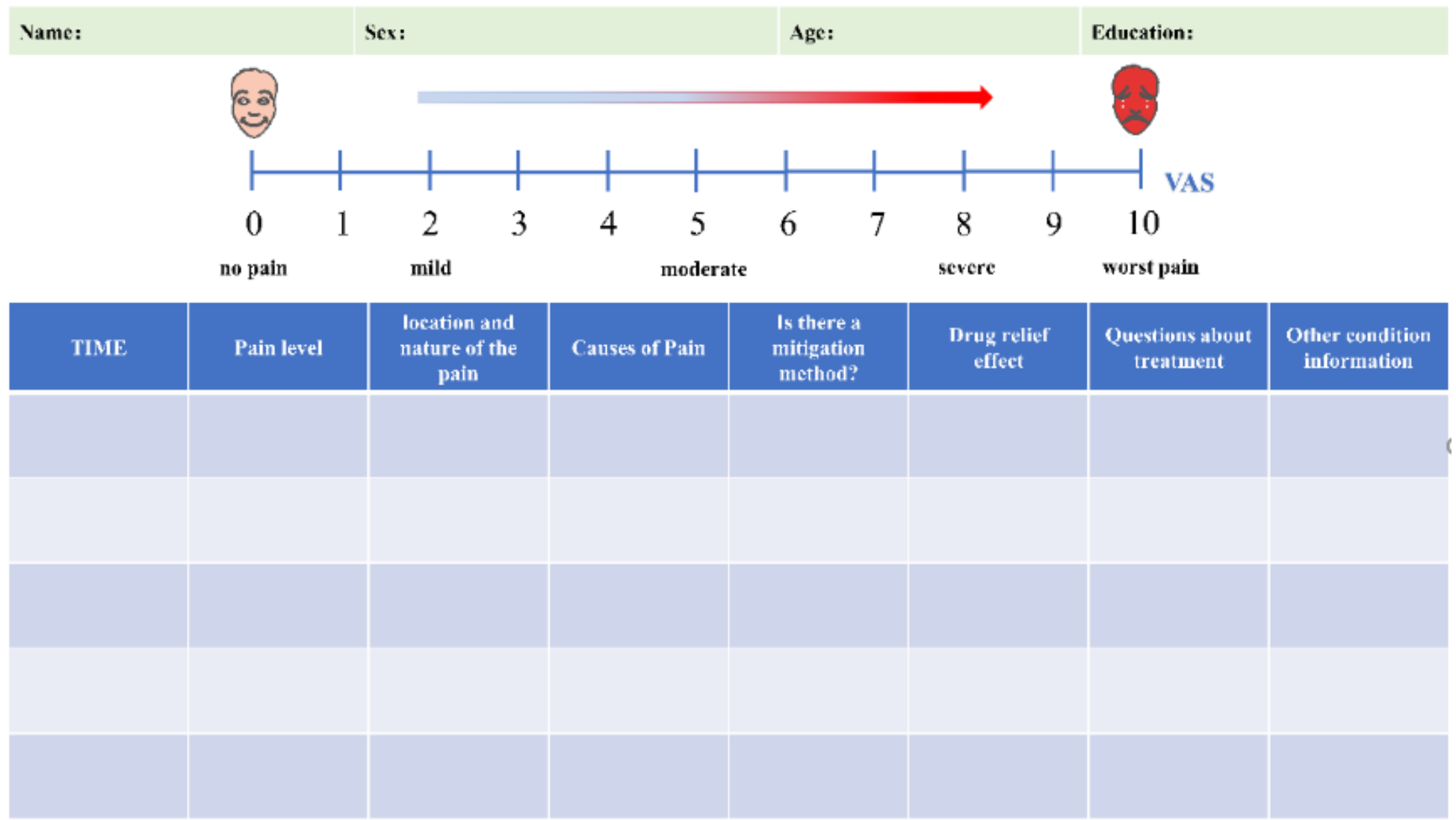

NOTE: Dear patient, please evaluate your pain according to the above VAS, and record your pain and illness information in time when the pain occurs(Accurate to the minute). When you need urgent help, don't be better than calling us, don't delay your illness because of the diary! If the form is not enough, please tell us in time, we will send a new diary form to your ward. Thank you for your cooperation!

Nurse in charge:

Table.2 Demographic and medical characteristics of diary group and control group $(n=119)$ 


\begin{tabular}{|lll|}
\hline & Control group $(\mathrm{n}=59)$ & Diary group $(\mathrm{n}=60)$ \\
\hline Age(year) (SD) & $47(14)$ & $48(12)$ \\
\hline Sex(F/M) & $40 / 19$ & $42 / 18$ \\
\hline Education level & & \\
\hline None & $0(0.0 \%)$ & $1(2.7 \%)$ \\
\hline Low & $8(27.1 \%)$ & $12(20.0 \%)$ \\
\hline Middle & $27(45.8 \%)$ & $29(48.3 \%)$ \\
\hline High & $16(27.1 \%)$ & $18(30.0 \%)$ \\
\hline Pain location & & \\
\hline Sternum & $44(74.6 \%)$ & $46(76.7 \%)$ \\
\hline Limbs & $11(18.6 \%)$ & $10(16.7 \%)$ \\
\hline Head and face & $4(6.8 \%)$ & $3(5.0 \%)$ \\
\hline Other & $0(0.0 \%)$ & $1(1.6 \%)$ \\
\hline
\end{tabular}

Note: F=Female; $M=$ Male

Table.3 comparison of Thai Brief Pain inventory between dairy group and control group

\begin{tabular}{|c|c|c|c|}
\hline Items & Control group Mean (SD) & Diary group Mean (SD) & $P$-value \\
\hline Maximum pain $24 \mathrm{~h}$ & $6.2(1.7)$ & $6.1(2.1)$ & 0.71 \\
\hline Minimum pain $24 \mathrm{~h}$ & $3,2(2.1)$ & $3.3(2.5)$ & 0.78 \\
\hline Average pain & $4.6(1.0)$ & $3.5(1.9)$ & $0.03^{\star}$ \\
\hline Pain now & $4.9(0.9)$ & $5.3(1.2)$ & 0.55 \\
\hline Treatment relief pain & $4.2(1.5)$ & $4.4(1.9)$ & 0.61 \\
\hline Activity & $5(2.5)$ & $4.8(2.9)$ & 0.42 \\
\hline Mood & $5.1(2.0)$ & $4.2(2.7)$ & $0.02^{*}$ \\
\hline Walking & $4.6(1.1)$ & $4.6(1.6)$ & 0.83 \\
\hline Sleep & $6.1(2.1)$ & $4.4(2.4)$ & $₫ 0.001^{*}$ \\
\hline Enjoyment of life & $5.1(2.9)$ & $4.4(3.0)$ & $0.02^{\star}$ \\
\hline
\end{tabular}


Note: $\mathrm{h}=$ hours; ${ }^{\star} P \otimes 0.05$ indicates comparison with control group. A higher score indicates a worse situation.

\section{Figures}

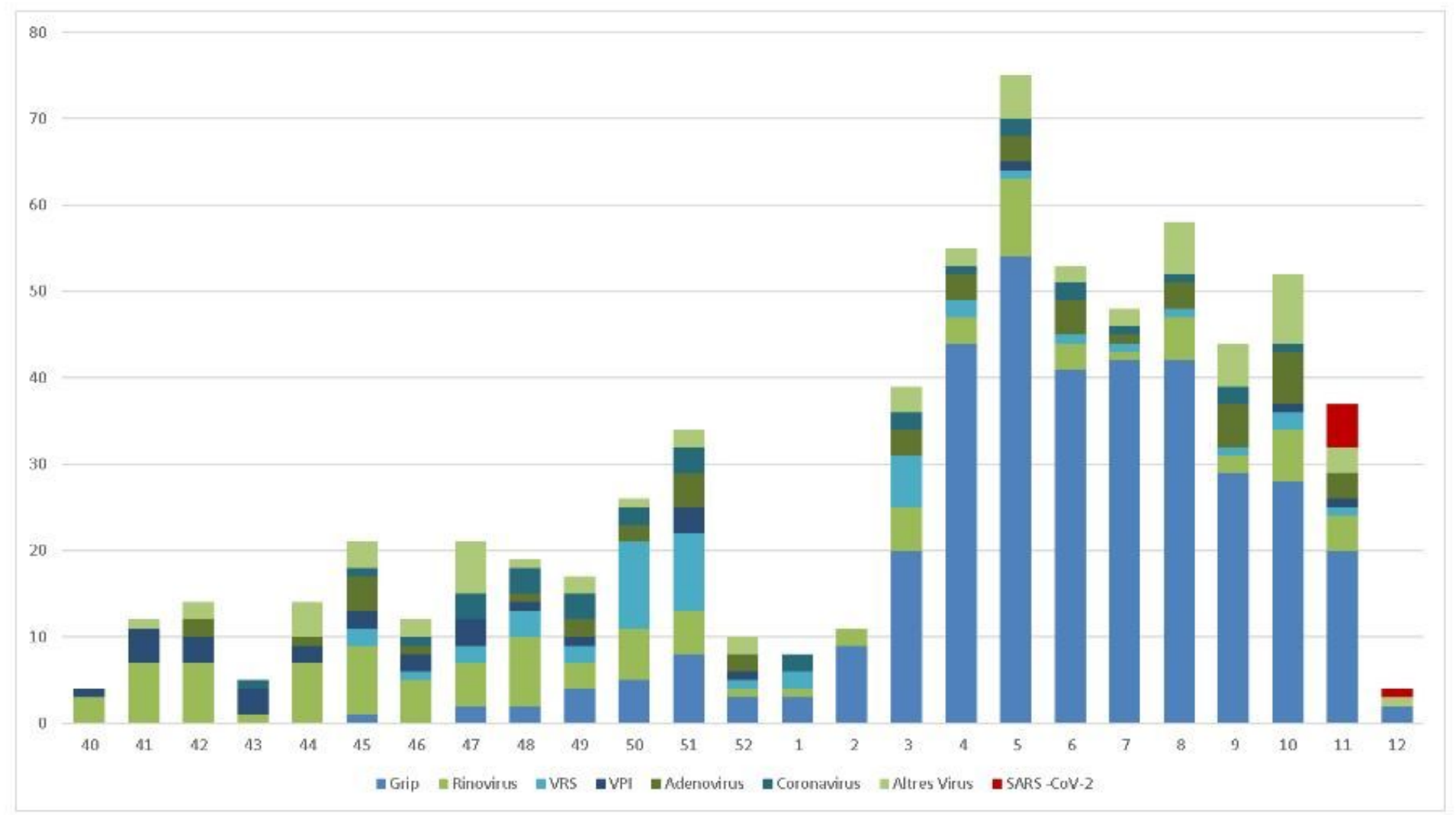

Figure 1

Patient flow diagram (Including the number of people finally included in the analysis). 


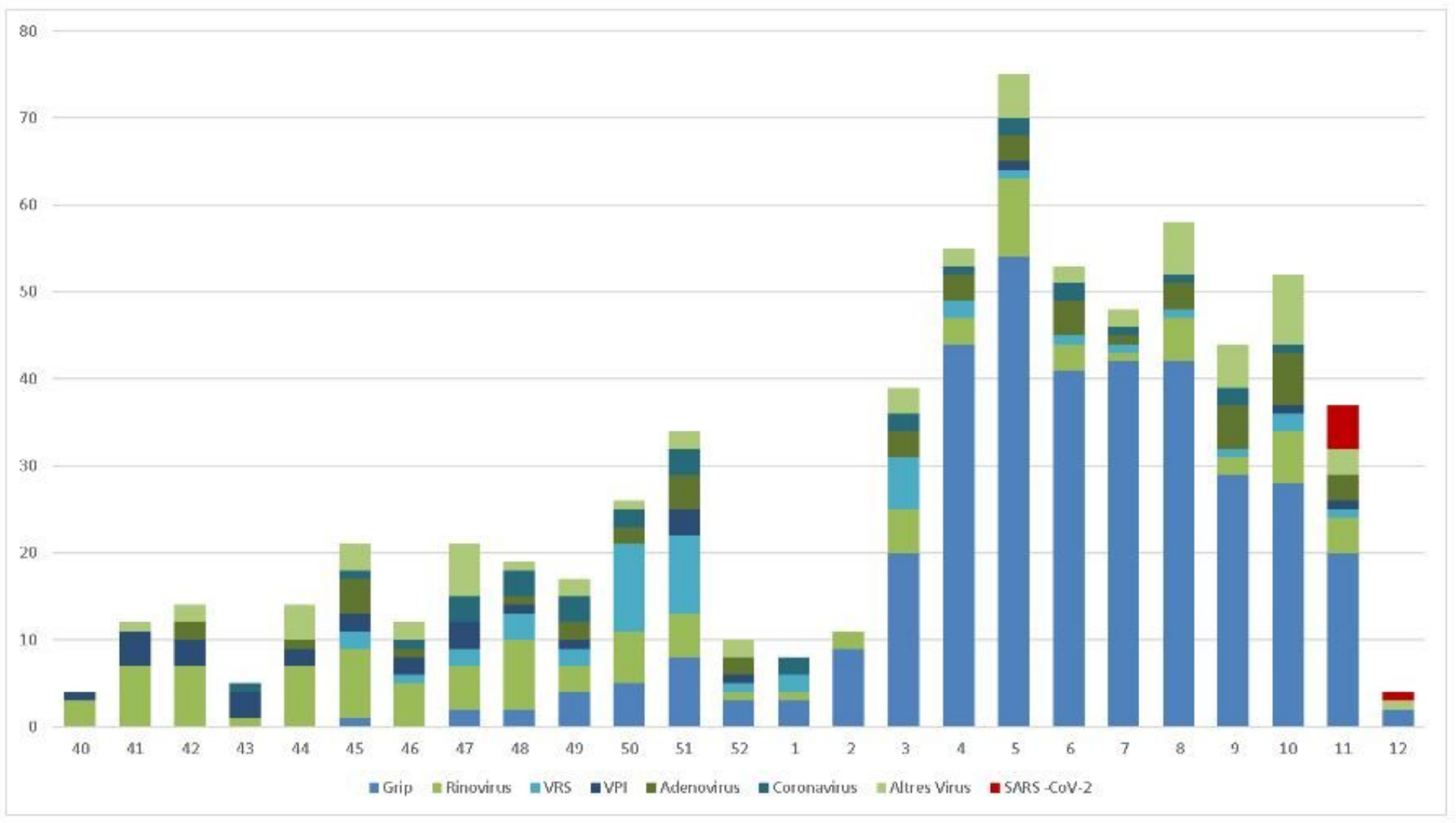

Figure 1

Patient flow diagram (Including the number of people finally included in the analysis). 


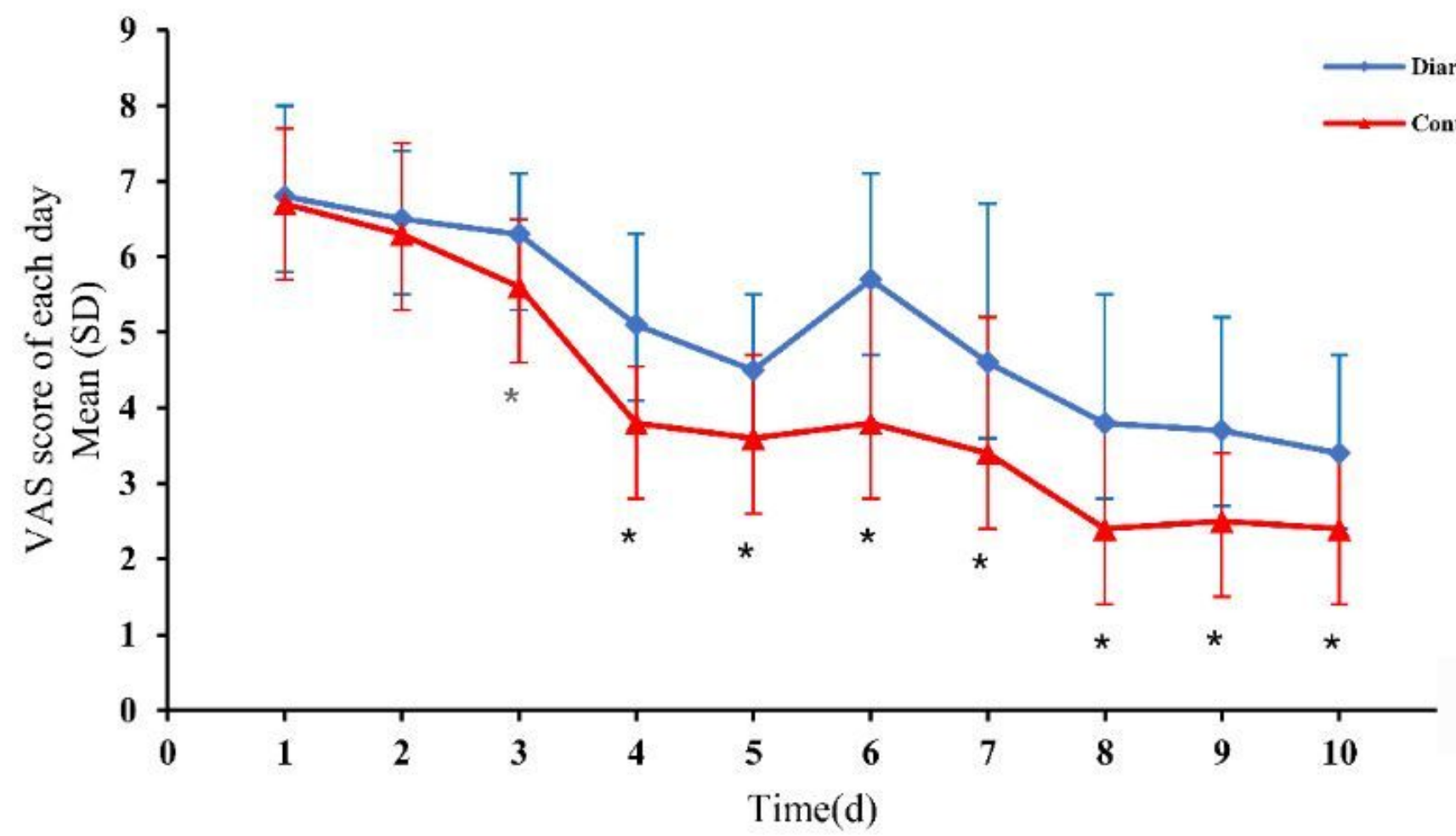

(1200dpi)

Figure 2

Changes in patients' VAS scores for 10 consecutive days. pain levels ranging from 0 to 10; 0 means no pain and 10 means unbearable pain. (ANOVA, ${ }^{\star} \mathrm{P} \otimes 0.05$ indicates comparison with control group) 


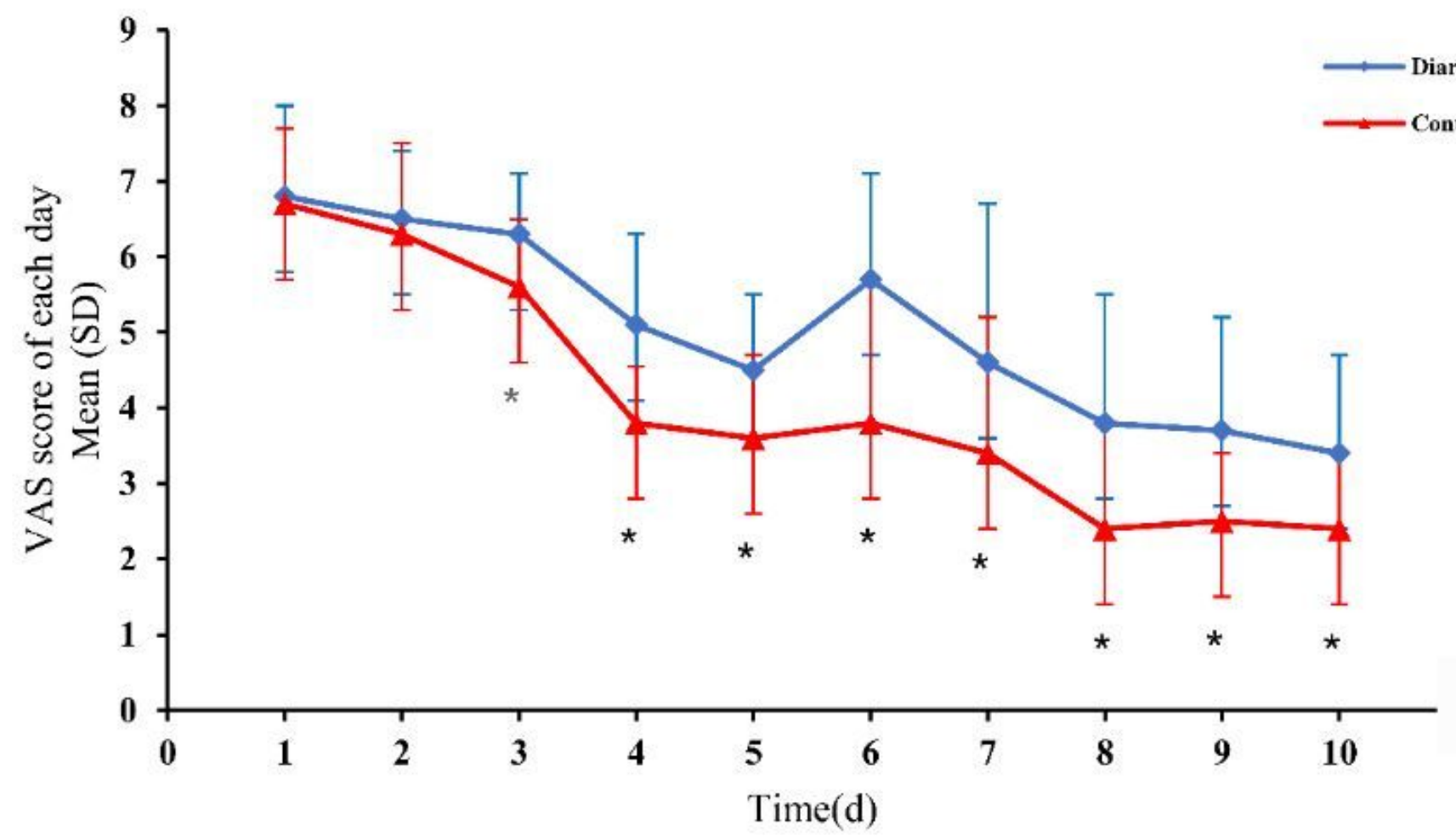

(1200dpi)

Figure 2

Changes in patients' VAS scores for 10 consecutive days. pain levels ranging from 0 to 10; 0 means no pain and 10 means unbearable pain. (ANOVA, ${ }^{\star} \mathrm{P} \otimes 0.05$ indicates comparison with control group) 
A

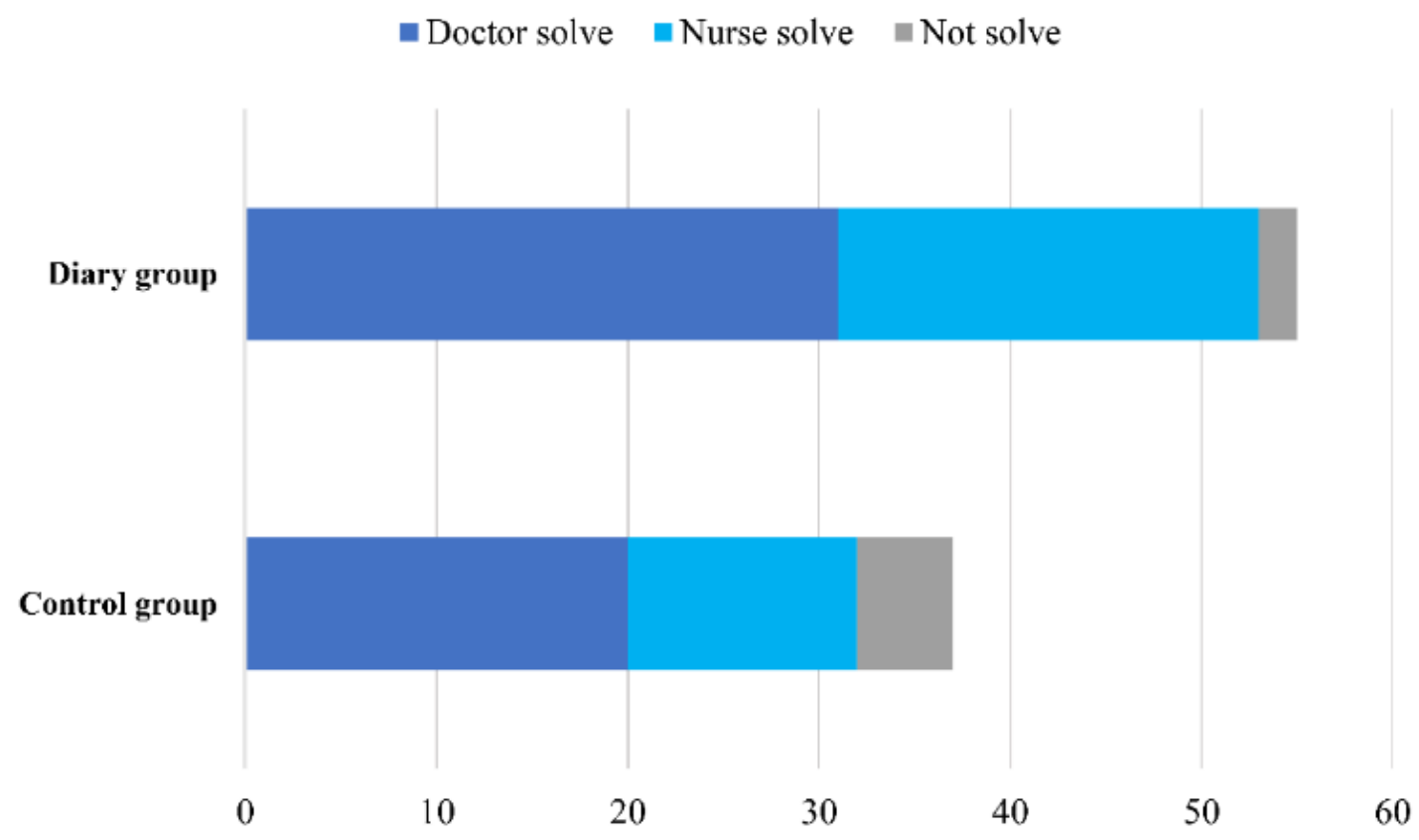

B

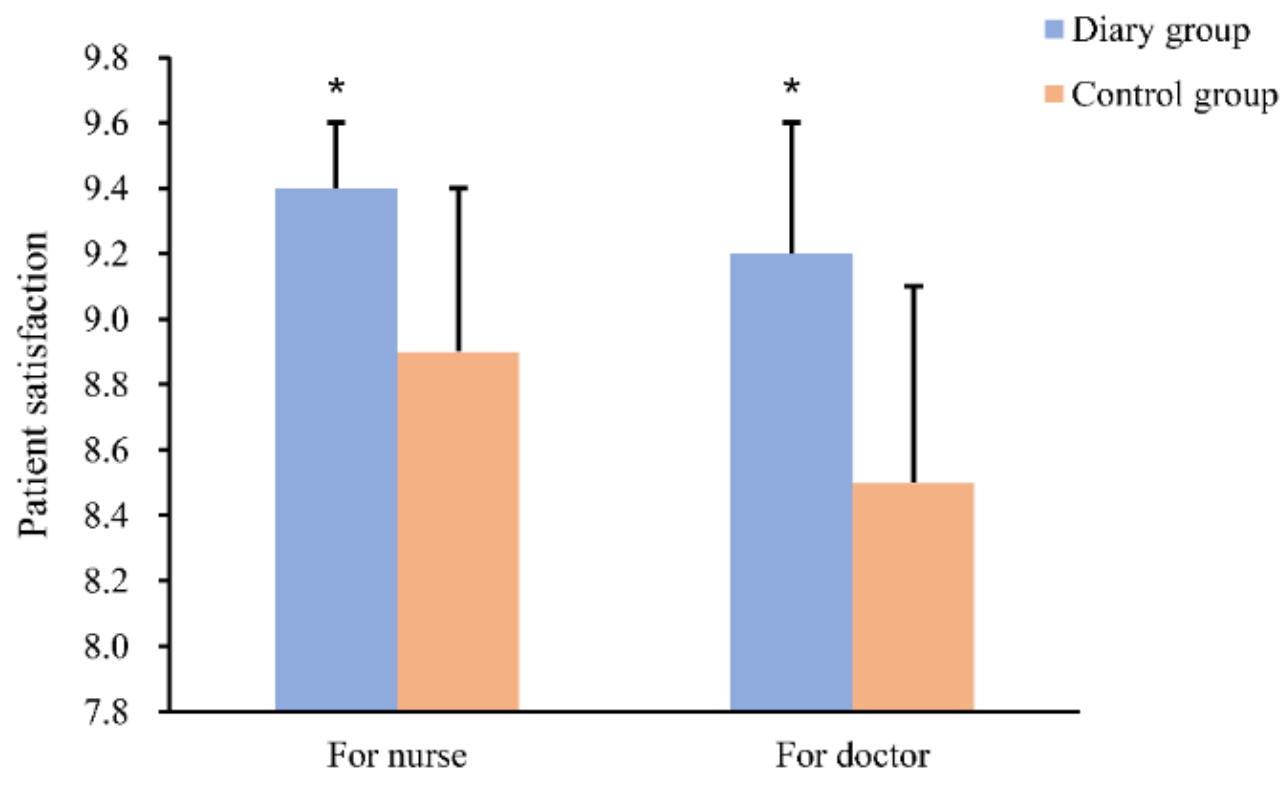

(1200dpi)

Figure 3

(A) Number of patient information (Student, *P $₫ 0.05$ indicates comparison with control group);(B) Patient satisfaction with doctors and nurses(Student, ${ }^{\star} \mathrm{P} \otimes 0.05$ indicates comparison with control group). 
A

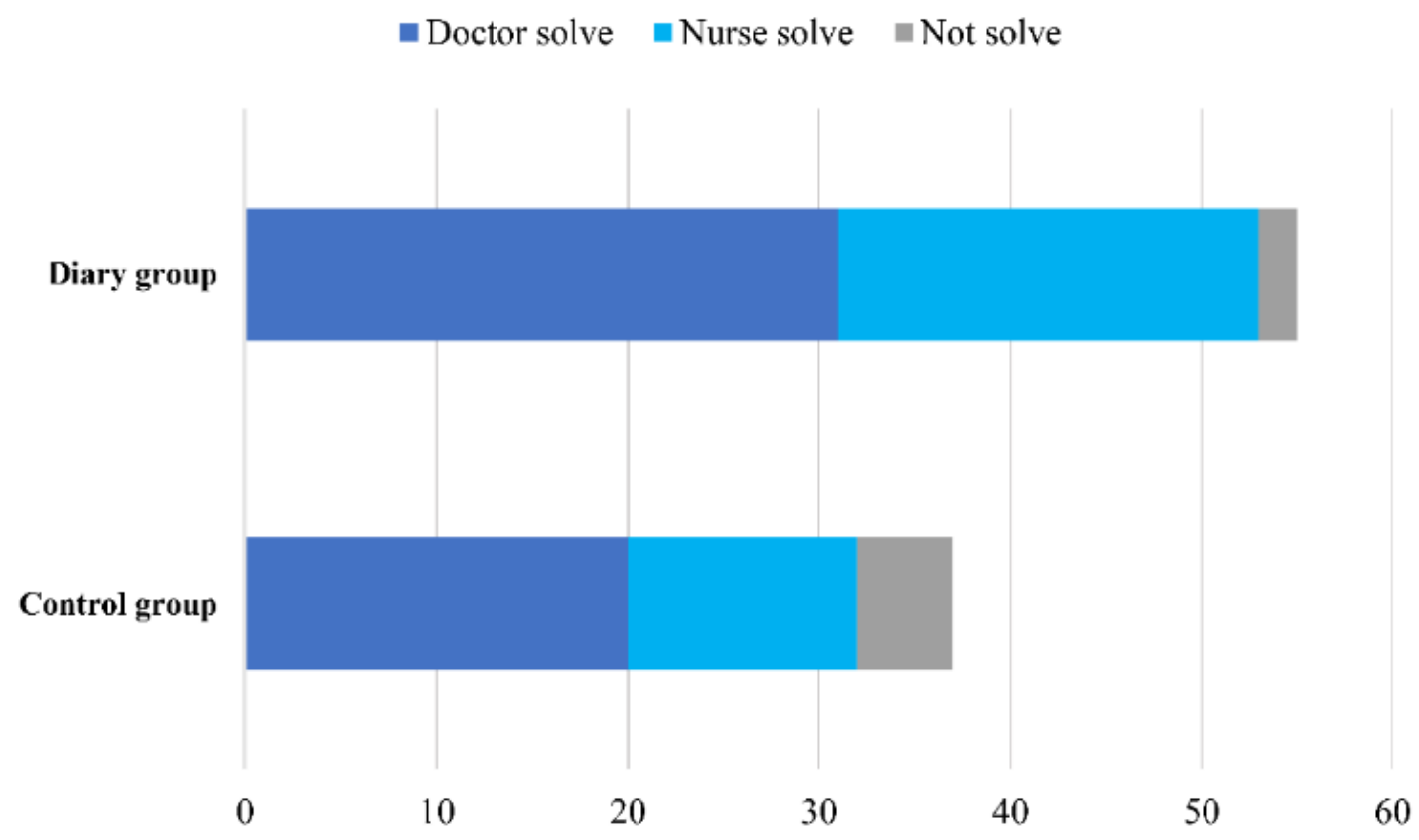

B

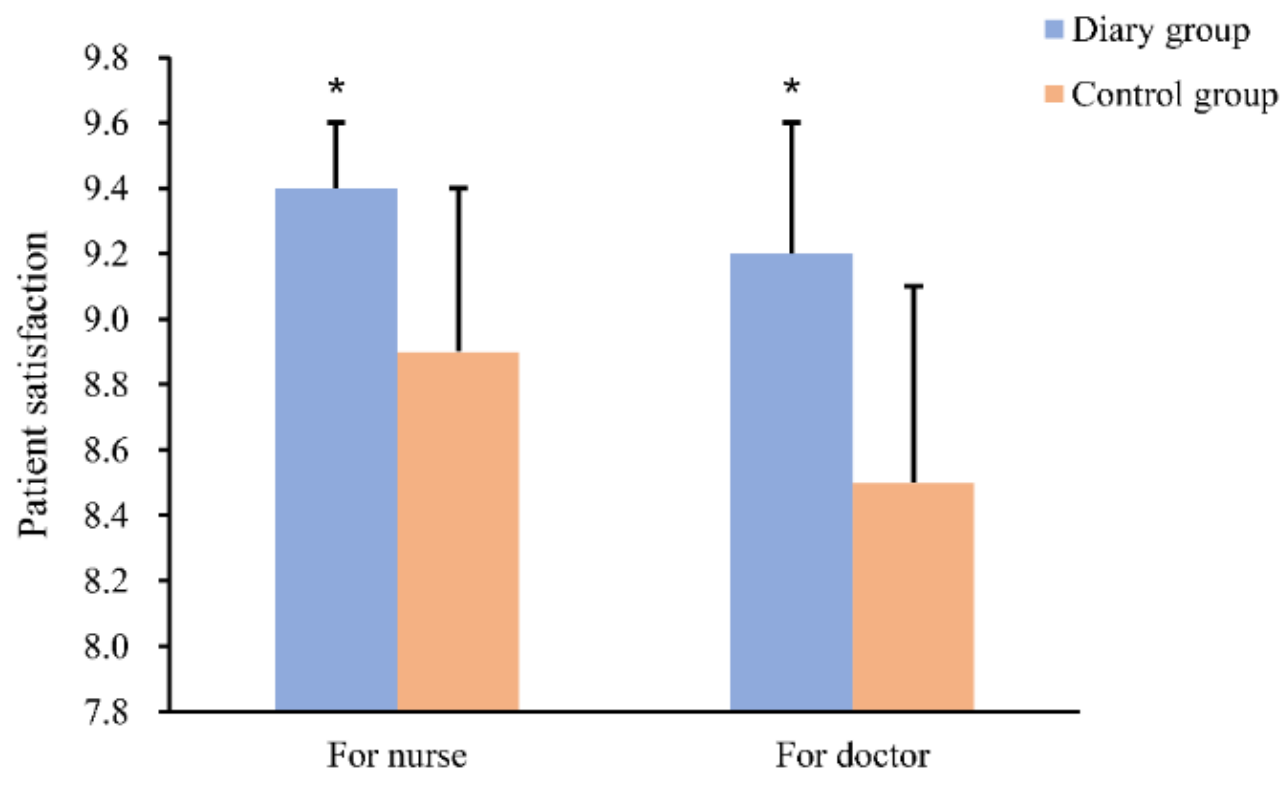

(1200dpi)

Figure 3

(A) Number of patient information (Student, *P $₫ 0.05$ indicates comparison with control group);(B) Patient satisfaction with doctors and nurses(Student, ${ }^{\star} \mathrm{P} \otimes 0.05$ indicates comparison with control group). 